\title{
A Holistic View of Polymer Aggregate
}

Polymers are everywhere. The living kingdom is full of natural polymers or biological macromolecules (e.g., polysaccharides, polynucleotides and proteins), while the modern society is alive with synthetic polymers or manmade macromolecules (e.g., plastics, fibers and rubbers). Although almost all the polymers are solid aggregates under ambient conditions, people have spent tremendous efforts to study their dilute solutions where the polymers exist as single molecular species. It is generally believed that understanding a molecule will help understand its aggregate, because a molecule, as defined by MerriamWebster, is "the smallest particle of a substance that retains all the properties of the substance". This reductionism approach has worked well and harvested much success. Useful information on and insight into structureproperty relationships have gained at the molecular level, resulting in the consensus that molecular behaviors determine materials properties.

Although popular, this reductionary epistemology is not always right. There exist many antireductionism systems. It is well known, for example, that many luminophores are emissive as molecules in the dilute solutions but do not luminesce as aggregates in the solid state, a photophysical phenomenon often referred to as aggregation-caused quenching (ACQ). The ACQ effect has posed a knotty hurdle to the development of real-world optoelectronic devices, such as the fabrication of light-emitting diodes from conjugated polymers. On the other hand, some luminogenic molecules do not luminesce but their aggregates emit efficiently, an anti-ACQ phenomenon known as aggregation-induced emission (AIE). ${ }^{[1]}$ The ACQ and AIE effects teach us that an aggregate is not necessarily a linear addition of its molecular components and that the properties of the former are not the simple extrapolations from those of the latter. In other words, a whole can contain the properties that cannot be discovered and comprehended through the analysis of its parts. Aggregate thus deserves to be studied as a separate entity in its own right. Aggregate science or aggregology study is anticipated to open new avenues to many attractive possibilities and generate much excitement in the exploration of exotic effects and new properties unique to an aggregate but not observable in its molecular systems. ${ }^{[2]}$

As mentioned above, most polymers are solid aggregates and are thus ideal objects of aggregology study. Indeed, immediately after the coinage of the AIE concept from the study of a small molecule system, the AIE re- search was quickly extended into big polymer systems. The booming development in the area has produced a large variety of polymeric AIE luminogens (AIEgens). The AIE polymers exhibited distinct materials properties and have found an array of high-tech applications in such diverse areas as optoelectronics, chemosensing, bioimaging and theranostics. ${ }^{[3]}$ Utilizing the working mechanism of restriction of intramolecular motions, ${ }^{[4]}$ researchers have used AlEgens to visualize the invisible structures and monitor the transition processes in macromolecular systems, such as crystal packing, domain boundary, chain alignment, blend morphology, microphase separation, segmental motion, glass transition, polarity change, sol-gel transition, living assembling, and polymerization reaction. ${ }^{[5]}$ In this short Editorial, I will briefly discuss two examples of polymer aggregate systems from the holistic viewpoint: one involving a $0 \rightarrow 1$ change (from nothing to something) and another concerning a $-1 \rightarrow+1$ transition (complete quality reversal), both enabled by aggregation processes.

The first example is the clusteroluminescence from the aggregates of $\pi$-electronically nonconjugated polymers. During the course of our AIE study, we observed emissions from photoexcited microparticles of poly[(maleic anhydride)-alt-(vinyl acetate)], a copolymer completely void of aromatic rings. This observation surprised us, as the modern organic electronics theory teaches that nonconjugated molecules do not luminesce. We examined a large number of nonconjugated polymers and found that the photophysical phenomenon was general. Many nonconjugated synthetic and natural polymers (polyacrylics, polyesters, polyamides, carbohydrates, proteins, DNAs, etc.) are photoluminescent in the aggregate state but nonemissive in the solution state. This is a nonconjugated version of AIE system, which can hardly be deciphered through a reductionism approach, as the molecular species of the polymers are nonluminescent. Holistically, we figured out that the phenomenon was essentially the clusterizationtriggered emission accompanying the polymer aggregation process. Although the macromolecules are $\pi$-electronically nonconjugated, they contain electronegative heteroatoms carrying lone-pair electrons $(\mathrm{O}, \mathrm{N}, \mathrm{S}, \mathrm{P}$, etc.). When the macromolecular chains are aggregated, the heteroatoms are clustered together via through-space interactions. ${ }^{[6]}$ The resultant clusteroluminogens can be photoexcited, thus making the polymers luminescent in the aggregate state. 
The example above demonstrates the ready generation of an entirely new property $(0 \rightarrow 1)$ by a simple aggregation process without changing molecular structure. The example below concerns the complete reversal in property $(-1 \rightarrow+1)$ upon aggregate formation. Poly( $N$-isopropylacrylamide) is known to undergo a dissolution-precipitation or sol-gel transition at a lower critical solution temperature. Employing a polarity-sensitive AIEgen, we proved that the transition was associated with a polarity change from molecular hydrophilicity (solubility in water) to aggregate hydrophobicity (immiscibility with water). ${ }^{[7]}$ Here again, the property change is induced by the physical aggregation without altering the chemical structure. Such transitions have been observed in many polymers, e.g., poly(ethylene glycol) and its block copolymer with poly(propylene glycol) as well as its supramolecular complex with $a$-cyclodextrin. The sol-gel transition is also common in living systems: many higher-order biological ensembles (cells, tissues, organs, etc.) are hydrophobic hierarchical aggregates of hydrophilic biomacromolecules (polysaccharides, polypeptides, etc.). In some sense, the structure-property relationship study at the aggregate level may hold the key to deciphering mysterious biological structures and processes.

Aggregation can make the impossible (at molecular level) possible (at aggregate level). This fact itself is antireductive, revealing the imperfection of molecular science. It is very difficult, if not impossible, to tackle all the issues involved in the aggregation processes and associated structures and properties from a pure reductionism approach. This calls for a paradigm shift from reductionism to holism. Viewing the aggregate as a whole will help solve the problems hard to handle by just analyzing its molecular parts. The insights gained from the aggregology study in polymer aggregates is anticipated to facilitate this paradigm shift, bringing scientific research to the systems at higher levels with greater complexity.

\section{REFERENCES}

1 Luo, J.; Xie, Z.; Lam, J. W. Y.; Cheng, L.; Chen, H.; Qiu, C.; Kwok, H. S.; Zhan, X.; Liu, Y.; Zhu, D.; Tang, B. Z. Aggregation-induced emission of 1-methyl-1,2,3,4,5-pentaphenylsilole. Chem. Commun. 2001, 1740-1741.

2 Tang, B. Z. Aggregology: exploration and innovation at aggregate level. Aggregate 2020, 1, 4-5.

$3 \mathrm{Hu}, \mathrm{R}$.; Qin, A.; Tang, B. Z. AlE polymers: synthesis and applications. Prog. Polym. Sci. 2020, 100, 101176.

4 Tu, Y.; Zhao, Z.; Lam, J. W. Y.; Tang, B. Z. Mechanistic connotations of restriction of intramolecular motions (RIM). Nat. Sci. Rev. 2021, 8, nwaa260.

5 Liu, S.; Tang, B. Z. Aggregation-induced emission: a novel approach to studying polymer science. Acta Polymerica Sinica (in Chinese) 2021, 52, 456-466.

6 Zhang, H.; Tang, B. Z. Through-space interactions in clusteroluminescence. JACS Au 2021, 1, 1805-1814.

7 Hu, Y.; Barbier, L.; Li, Z.; Ji, X.; Blay, H. L.; Hourdet, D.; Sanson, N.; Lam, J. W. Y.; Marcellan, A.; Tang, B. Z. Hydrophilicityhydrophobicity transformation, thermoresponsive morphomechanics and crack multifurcation revealed by aiegens in mechanically strong hydrogels. Adv. Mater. 2021, 33, 2101500.

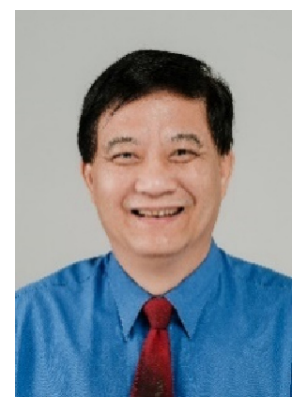

Ben Zhong Tang

Shenzhen Institute of Aggregate Science and Technology, School of Science and Engineering,

The Chinese University of Hong Kong, Shenzhen, China; Email: tangbenz@cuhk.edu.cn

\section{NOTES}

The views expressed in this editorial are those of the author. 